\title{
Report from the International Pancreas Transplant Registry
}

\author{
D. E. R. Sutherland \\ Department of Surgery, University of Minnesota Medical School, Minneapolis, Minnesota, USA
}

Summary. From December, 1966 through 1990, 3082 pancreas transplants were reported to the International Registry. A detailed analysis was performed on the 2087 transplants in the 1986-90 data base; during this time the overall one year recipient and graft functional survival rates were $89 \%$ and $62 \%$. Univariate analyses showed graft survival rates to be significantly higher with bladder drainage than with other duct management techniques, with a simultaneous kidney transplant than without, and with preservation in UW than other solutions. Storage up to 30 hours did not adversely effect results. Graft survival rates were significantly higher in North America than Europe, a discrepancy that persisted in a Cox proportional hazard analysis that also included duct management, recipient category, HLADR mismatching, immunosuppression with anti- $T$ cell agents, preservation solution and duration, and year of transplant as the other variables; three were identified to have a significant $(\mathrm{p}<0.05)$ impact on the relative risk (RR) of graft loss: 1) Recipient category, with an RR of 0.43 when placed simultaneously with a kidney; 2) Year, with an RR of 0.76 for transplants performed in 1989-90 versus 1986-88; and 3) Location, with an RR of 0.75 for transplants in North America versus Europe. The technical failure rate was significantly lower in North America than Europe, but this was not an explanation for the differences in outcome, since the same factors sorted out as significant in a Cox proportional hazard analysis of technically successful cases only. Furthermore, logistic regression analysis showed retransplantation, duct management, recipient category, preservation solution, and storage time to significantly influence the technical failure rate. The risk of technical failure was lowest for primary, bladder-drained, simultaneous pancreas/kidney, < 12 hour UW stored grafts; year and location of transplant were not significant factors. Pancreas transplant results continue to improve and are in the range of those for other solid organs.
Key words: Pancreas - Transplant - Registry

\section{Introduction}

In this report from the International Pancreas Transplant Registry, the results in five eras for all cases reported worldwide from December 17, 1966 to October 21,1990 are given. The results according to multiple variables are also summarized for cases reported since January 1, 1986. Finally, a comparison of outcome in North American, Europe and other locations is made for 1986-90 cases.

\section{Methods}

Actuarial patient and graft survival rates were calculated on a CDC Cyber computer using SPSS software, as previously described (Sutherland et al. 1989). Pancreas graft function was defined as insulin-independence (virtually all recipients were Type I diabetics), and death with a functioning graft was considered a graft failure. Analyses were done of all cases as well as of technically successful (TS) cases, with technical failure (TF) defined as grafts that underwent thrombosis, or were removed for local infection, bleeding, anastomotic leaks or other non-immunological problems. The test of Gehan (1965) was used to compare groups on univariate survival analyses. The Chi Square test was used to compare the incidence of certain events. The Cox (1972) proportional hazard technique with a backward and forward stepwise model on BMDP sof tware was used to determine the importance of several variables on relative risk (RR) for graft loss. Logistic regression analysis was performed to determine the factors that had an impact on the technical failure rate (Lemeshow and Hosmer 1984). P-values $<0.05$ were considered significant.

Number of Transplants and World Results by Era Since 1966

As of October 21, 1990, the cutoff date for inclusion in the analyses, 2871 pancreas transplants (2703 primary, 168 regrafts) had been reported to the International Pancreas Transplant Registry, including 1663 in North 
America, 1158 in Europe, and 50 in other locations (Figure 1).

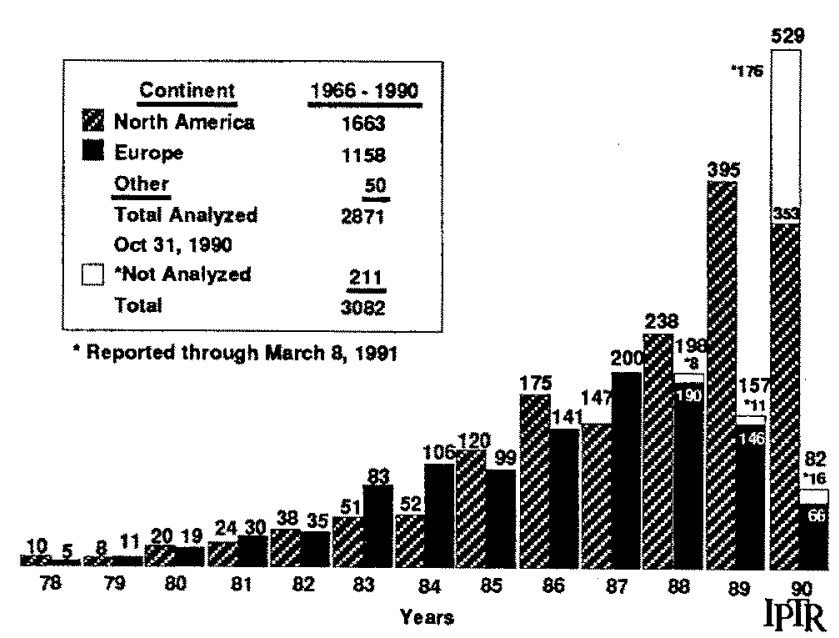

Fig.1. Number of transplants reported by year according to location. For years 1966-77 (not shown) 64 cases were reported ( 32 in North America, 27 in Europe, 5 elsewhere).

Two-hundred-eleven additional pre- 1991 cases were reported after this date (up to March 8, 1991), 8 for 1988 and 11 for 1989 (all European) and 192 for 1990 (176 North America, 16 Europe), bringing the known total in 1990 to 619 and the world total since 1966 to 3082 (1839 North America, 1193 Europe, and 50 other). One-hundred-forty-four institutions have reported pancreas transplant cases to the Registry since 1966 , including 80 in North America, 48 in Europe, and 16 elsewhere.

Recipient and pancreas graft survival rates have improved with time (Figure 2)

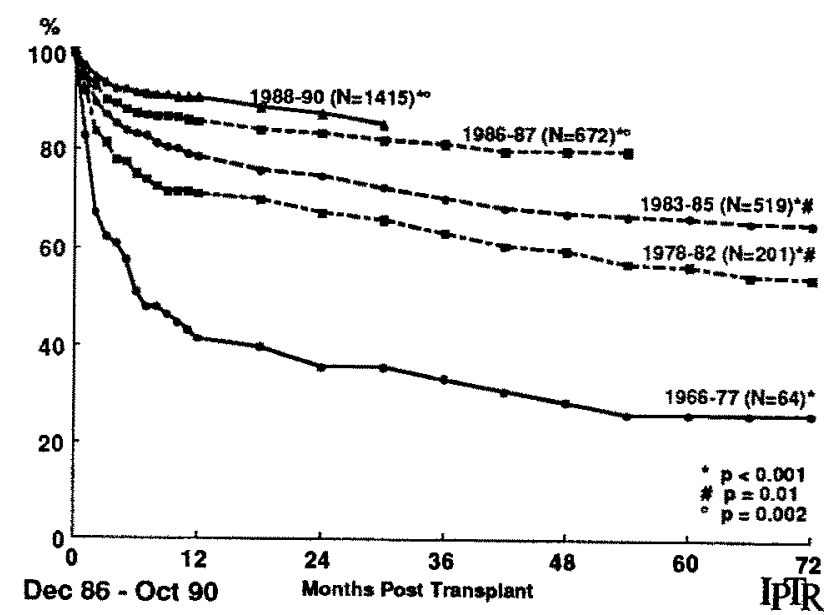

Fig.2A. Patient survival rates by world era

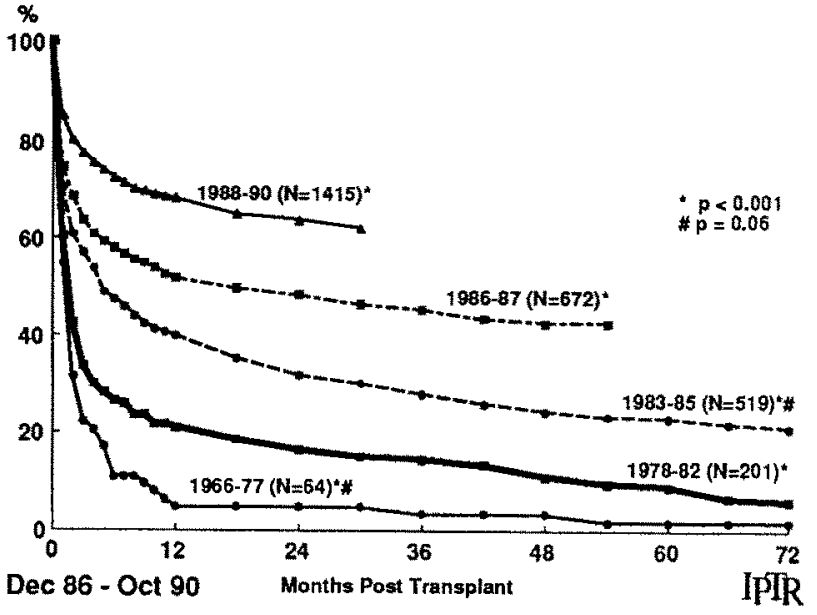

Fig.2B. Pancreas graft function survival rates by world era

In five consecutive eras, $1966-77(n=64) 1978-82(n=201)$, $1983-85(n=519), 1986-87(n=672)$, and $1988-90(n=1415)$, one year patient survival rates were $41 \%, 71 \%, 79 \%, 86 \%$, and $91 \%$, and one year graft survival rates were $5 \%, 21 \%$, $40 \%, 52 \%$ and $68 \%$, respectively ( $\leq 50.002$ for all comparisons after 1977).

The increases in graft survival rates between each era are of similar magnitudes. There is room for further improvement, but the current results of pancreas transplantation are in the range of other solid organ transplants.

One of the reasons for the improvement in graft survival rates is the reduction in technical failure rates $(13 \%$ for $1988-90$ cases versus $23 \%$ for $1986-87$ cases, $\mathrm{p}<0.001$ ). However, this is not the entire explanation for the improvement in results, since even for TS cases, the graft survival rates were significantly higher $(\mathrm{p}<0.001)$ for $1988-90(\mathrm{n}=1229)$ than for $1986-87(\mathrm{n}=515)$ cases, at one year being $79 \%$ and $63 \%$ respectively.

In order to have a sufficient number of cases with long-term follow-up in all possible subgroups, the last two eras were combined into a single period (1986-90) for the analyses described in the following section.

The overall one year patient and graft survival rates for 1986-90 cases $(n=2037)$ were $89 \%$ and $62 \%$. The overall technical failure rate was $16 \%$. The functional survival rate at one year for TS grafts $(n=1774)$ transplanted during $1986-90$ was $74 \%$.

Effects of Multiple Variables on Outcome in Analysis of World Data for 1986-1990

Of the 2087 pancreas transplants performed between January 1 , 1986 and October 21, 1990 that were available for analysis in the Registry data base, 1973 were primary and 114 were retransplants. At one year the graft functional survival rate was 
$64 \%$ forallprimarypancreastransplantsand $41 \%$ forall retransplants $(\mathrm{p}<0.001)$. The technical failure rate was $16 \%$ for primary and $28 \%$ for retransplant cases $(p<0.001)$. The lower technical failure rate was not the sole explanation for the superior results with primary transplants since even in the analysis of technically successful (TS) cases the functional survival rate was significantly higher $(p<0.01)$ for first grafts $(n=1662)$ than for regrafts $(n=82)$, at one year being $75 \%$ versus $57 \%$.

\section{Surgical Technique}

The three most common methods for pancreatic graft duct management during 1986-90 were bladder-drainage(BD, threefourths of cases), duct injection (DI) with a synthetic polymer (between one-sixth and one-seventh of cases), and intestinal drainage (ID, one-twelfth of cases).

Bladder-drainage $(n=1566)$, the most frequently used technique, was associated with a significantly higher $(\mathrm{p}<0.001)$ graft functional survival rate than either polymer injection $(n=323)$, or intestinal drainage $(n=174)$, at one year being $65 \%$ versus $56 \%$ and $52 \%$, respectively (Figure 3 ).

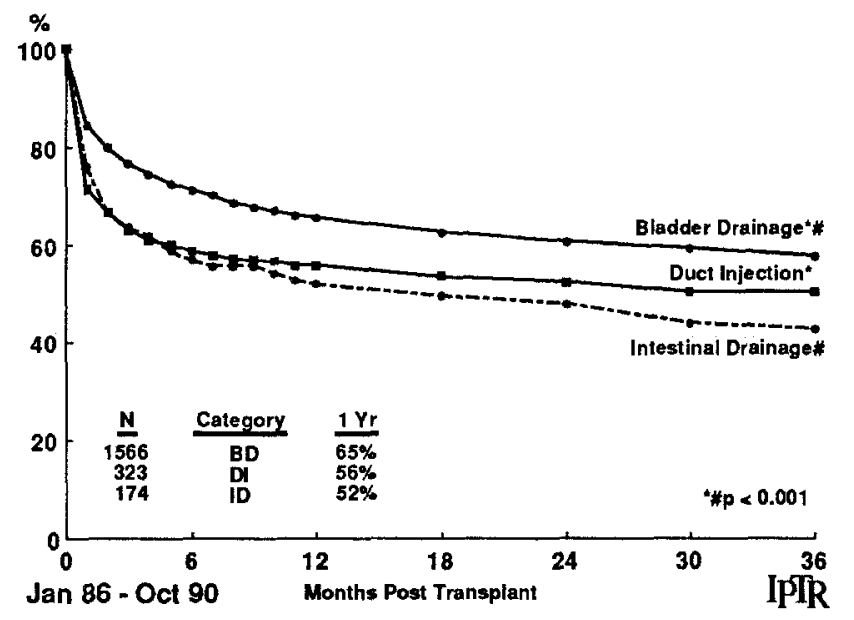

Fig.3. Pancreas graft functional survival rates according to methods of duct management for 1986-90 world cases

The technical failure rate was significantly lower $(p<0.001)$ with bladder-drainage (14\%) than with polymer injection (25\%) or intestinal drainage (23\%). The lower technical failure rate was not the sole explanation for superior results with the bladder-drainage tehnique, since even for TS cases $(n=1354)$ the graft functional survival rate was significantly higher $(p<0.04)$ in this category than in the duct injection $(n=242)$ or intestinal drained ( $n=133$ ) categories, at one year being $76 \%$ versus $72 \%$ and $66 \%$ respectively.

\section{Recipient Categories}

More than three-fourths of the cases reported during 1986-90 were simultaneous pancreas/kidney (SPK) transplants $(n=1644)$.
Slightly more than one-tenth were pancreas transplants after a previous kidney (PAK) transplant $(n=223)$, and slightly less were pancreas transplants alone (PTA, $n=212$ ) in non-uremic non-kidney recipients. The pancreas graft functional survival rate was singnificantly higher $(p<0.001)$ in the SPK than the PAK or PTA recipients, at one year being $68 \%$ versus $45 \%$, and 37\% (Figure 4).

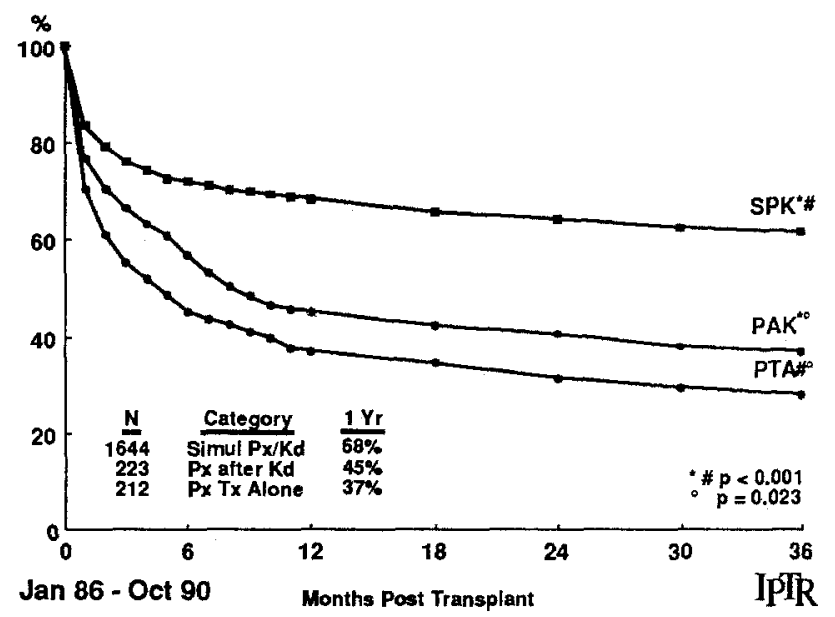

Fig.4. Pancreas graft functional survival rates according to recipient category for 1986-90 world cases

The technical failure rates in the SPK, PAK, andPTA categories were $15 \%, 19 \%$ and $27 \%$ ( $<<0.001$ for SPK and for PAK versus PTA). Again, the lower technical failure rate in the SPK cases is not the sole explanation for the better results. For technically successful (TS) cases, the pancreas graft functional rate was significantly higher $(p<0.001)$ in the SPK $(n=1402)$ than either the PAK $(n=180)$ or PTA $(n=155)$ categories, at one year being $80 \%$ versus $55 \%$ and $50 \%$. In contrast, patient survival rates were higher in recipients of solitary pancreas transplants, being 94\% at one year in PAK recipients of a pancreas after a kidney, and $91 \%$ in PTA recipients of a pancreas transplant alone, as compared to $88 \%$ in SPK recipients of a simultaneous pancreas and kidney transplant ( $\mathrm{p} \leq 0.05$ for PAK versus SPK). Patient survival rates were nearly identical in the analysis of TS cases, at one year being $94 \%, 91 \%$ and $90 \%$ in the respectivecategories ( $\mathrm{p}=\mathrm{ns}$ for all comparisons).

\section{Kidney Graft Survival in SPK Recipients}

The overall one year kidney graft survival in 1986-90 SPK recipients was $80 \%$. Loss of pancreas function with maintenance of kidney function was more common than loss of kidney function with maintenance of pancreas function, primarily because more pancreas grafts were lost for technical reasons. In SPK cases where the pancreas was a technical failure (TF) $(n=119)$ the kidney graft survival rate was $62 \%$ at one year. The one year kidney graft survival rate was $86 \%$ in SPK cases where the pancreas was technically successful $(n=835)(p<0.001$ versus TFcases). The pancreas and kidney graft survival rates werenot significantly different in the analysis of SPK cases where the 
pancreas was TS, in contrast to the results in the analysis of all cases. The risk of immunological loss is probably similar for both organs. In any event, for 1986-90 SPK recipients the probability of being alive at one year was $88 \%$, of being dialysis free was $80 \%$ and of being insulin-independent was $68 \%$, and there was little change in status over the next two years in this group (Figure 5).

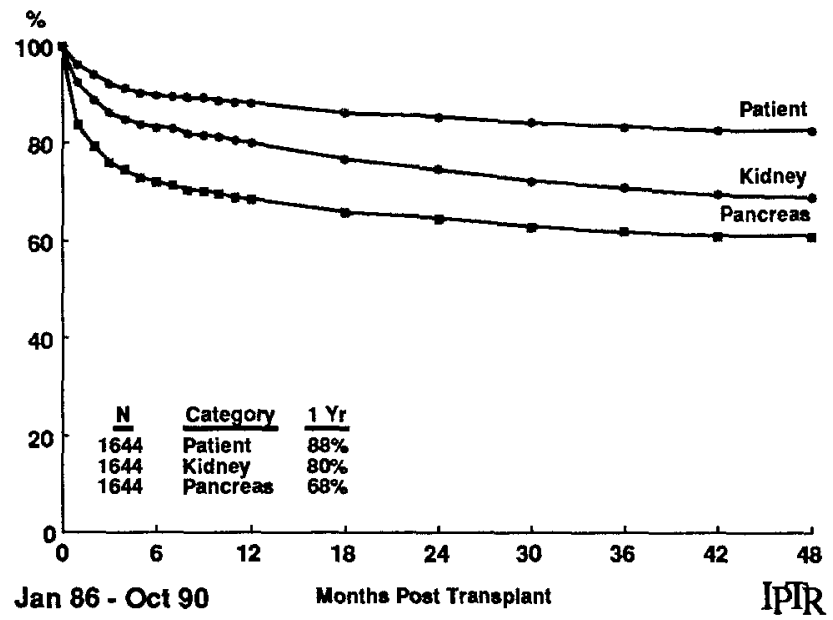

Fig.5. Patient, kidney and pancreas graft survival rates in 1986-90 world SPK cases

\section{HLA DR Matching and Mismatching}

HLA-DR antigen matching or mismatching was shown to significantly influence pancreas graft survival rates in previous Registry data (Squifflet et al 1988; Sutherland et al. 1990). The effect of DR matching is less strong in the current analysis of 1986-90 cases. The largest difference was seen in the analysis of pancreas transplants alone. For all PTA cases (regardless of duct management technique), the one year graft functional survival rate was $54 \%$ for recipients matched for $2 \mathrm{DR}$ antigens with a donor $(n=13)$, versus $44 \%$ for recipients matched for only $1 \mathrm{DR}$ antigen $(n=63)$, and $29 \%$ in recipients matched for 0 antigens ( $n=94$ ), with the 2 versus 0 DR mismatch being significant $(\mathrm{p}=0.015)$. In the overall PAK category, the differences were smaller, and not significant, with one year pancreas graft functional survival rates in the $2(\mathrm{n}=16), 1(\mathrm{n}=74)$, and $0(\mathrm{n}=89)$, HLA-DR matched groups of $53 \%, 51 \%$, and $39 \%$, respectively. In the overall SPK category, there were no differences at all, with one year pancreas graft functional survival rates of $69 \%$ for graft from donors matched for either $2(n=51), 1(n=547)$, and $0(n=806)$ HLA-DR antigens with the recipients. In the analysis of all TS cases, the differences in graft survival rates according to DR match were not significant in any of the recipient categories, with functional survival rates for 2, 1 , and $0 \mathrm{DR}$ matched grafts being $58 \%(n=12), 58 \%(n=118)$, and $41 \%(n=65)$ for PTA cases; $61 \%(n=14), 57 \%(n=62)$, and $51 \%(n=69)$ for PAK cases; and $87 \%(n=41), 82 \%(n=459)$, and $80 \%(n=698)$ for SPK cases.

Since pancreas graft outcome varies according to recipient category and duct management technique, the variables were controlled by determining the effect of HLA-DR matching with all of the possible duct management/recipient subcategories. Results within the recipient subcategory managed by the bladder-drainage (BD) technique are given in Figure 6. With BD, the one year functional survival rates for graft from donors matched for 2, 1, or $0 \mathrm{DR}$ antigens with the recipients were $55 \%(n=11), 47 \%(n=49)$, and $33 \%$ $(n=65)$ within the PTA subcategory (Figure 6A);

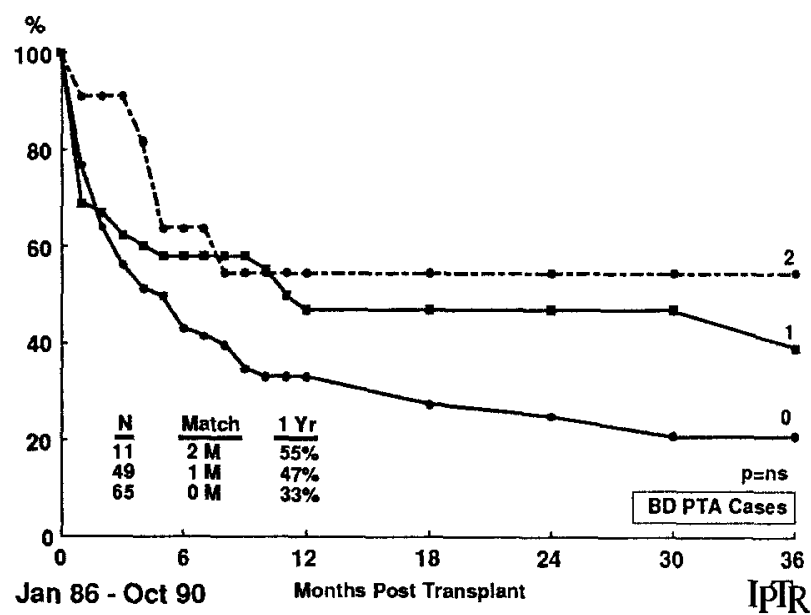

Fig.6A. Graft functional survival rates in 1986-90 world bladder drained pancreas transplants alone according to HLA-DR match

$58 \%(n=15), 51 \%(n=72)$, and $38 \%(n=78)$, within the PAK subcategory (Figure 6B);

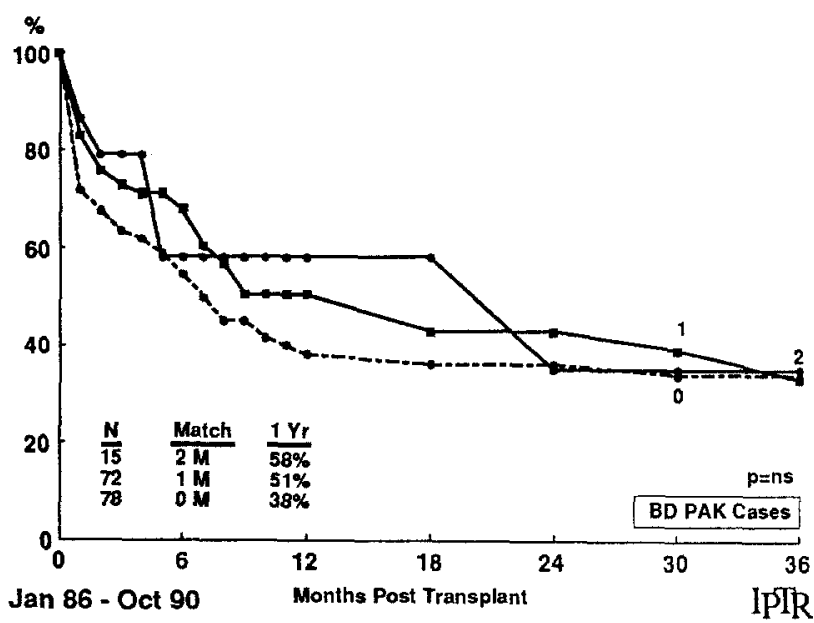

Fig.6B. Graft functional survival rates for 1986-90 world bladder-drained pancreas transplants after a kidney according to HLA-DR match 
and $77 \%(\mathrm{n}=37), 72 \%(\mathrm{n}=432)$, and $73 \%(\mathrm{n}=620)$ within the SPK category (Figure 6C).

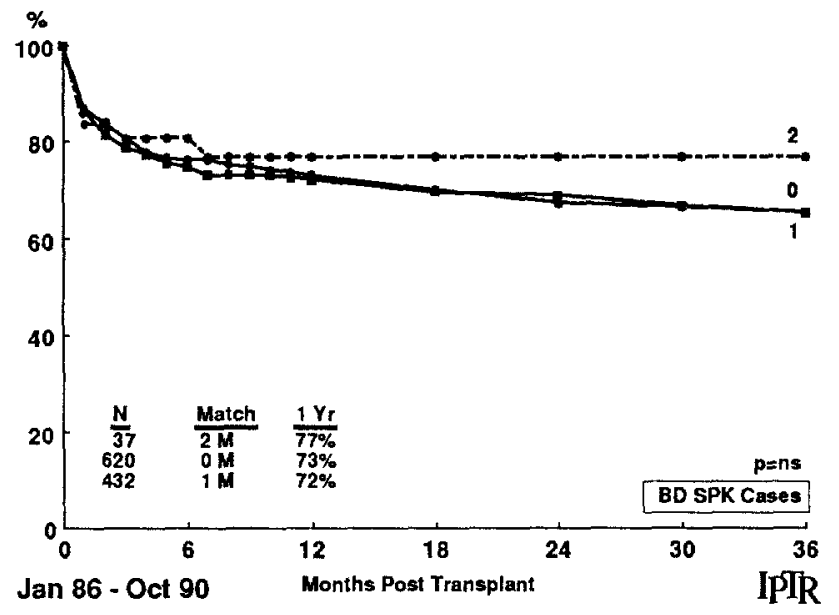

Fig.6C. Pancreas graft functional survival rates for 1986-90 world bladder-drained simultaneous kidney transplant cases according to HLA-DR match

In the corresponding analysis of TS $\mathrm{BD}$ cases by recipient categories, the one year functional survival rates for 2,1 , or $0 D R$ matched grafts were $60 \%(n=10), 58 \%(n=40)$, and $45 \%$ $(\mathrm{n}=48)$ within the PTA subcategory; $68 \%(\mathrm{n}=13), 56 \%(\mathrm{n}=61)$, and $49 \%(n=61)$ in the PAK subcategory; and $89 \%(n=32)$, $85 \%(\mathrm{n}=368)$, and $82 \%(\mathrm{n}=552)$ in the SPK subcategory.

Every comparison showed the graft survival rates to be progressively higher from 0 to 1 to $2 \mathrm{DR}$ matches, but none of the comparisons within the $\mathrm{BD}$ recipient subcategories were statistically significant. However, when the BD PTA and PAK subcategories were combined into a BD solitary pancreas transplant subcategory, a significant difference was observed, with one year functional survival rates of $55 \%$ with a 2 DR match $(n=26), 49 \%$ with a $1 D R$ match $(n=121)$, and $36 \%$ with a 0 DR match $(n=143)(p<0.05$ for the 1 versus the $0 D R$ match comparison). The same relative differences were seen in the analysis of TS solitary BD cases, but without statistical significance, with one year pancreas graft survival rates in the 2,1 , and 0 DR matched categories of $63 \%(n=23), 57 \%(n=101)$, and $47 \%(n=109)$.

The analyses of outcome according to HLA-DR mismatches mirrored the preceding analyses, with $0 \mathrm{DR}$ mismatches associated with higher graft survival rates than 2 DR mismatches, and $1 \mathrm{DR}$ mismatches generally being intermediate. Again, only an occasional comparison was statistically significant. The differences are small in the SPK category, and thus do not approach significance even with relatively large numbers in each matched category. The largest differences are seen in the PTA category, but here the number of cases is small. Thus, no conclusions about the effect on HLA-DR matching of pancreas graft outcome should be made at this time.

\section{Immunosuppression}

In previous analyses of Registry data, pancreas graft functional survival rates were compared for recipients who were treated with cyclosporine without azathioprine, azathioprine without cyclosporine, or cyclosporine and azathioprine together, and the rates were significantly higher in the combination group (Sutherland et al. 1989). Such an analysis has become irrelevant, since there are so few $(<15 \%)$ in the 1986-90 analysis who were not treated with both drugs. Thus, in the current analysis, the emphasis was placed on whether anti $\mathrm{T}$-cell agents were used prophylactically (either OKT3 [ $n=319]$ or ALG $[n=1025])$. The pancreas graft functional survival rates in both of these groups were significantly higher than in the group who received neither agent $(n=711)$, with one year graft survival rates being $71 \%, 63 \%$, and $59 \%$, respectively ( $\mathrm{p}=0.001$ for OKT3 and 0.038 for ALG versus the non anti-T-cell agent group, with the difference between the OKT3 and ALG group not being significant) (Figure 7).

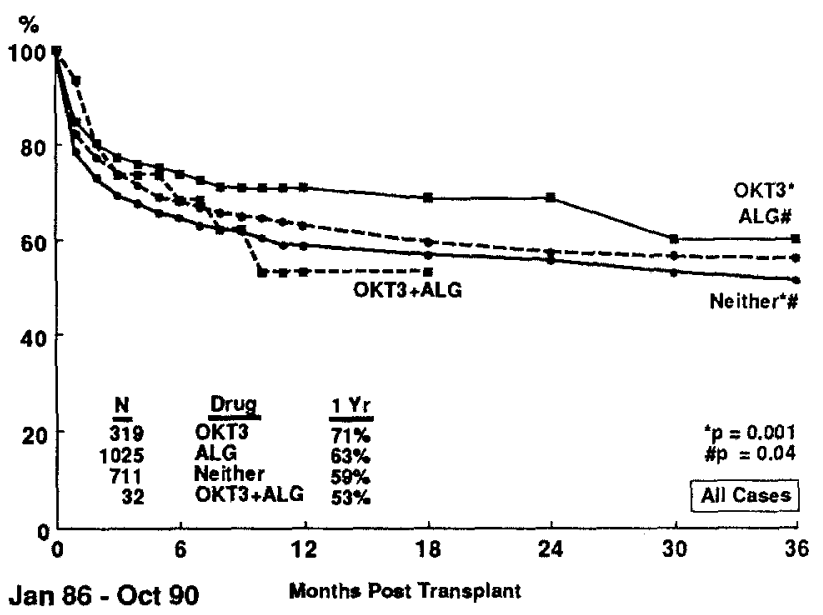

Fig.7. Pancreas functional survival rates for 1986-90 world cases according to use of anti-T cell antibody therapy for prophylactic immunosuppression

Whether the apparent beneficial effect of anti T-cell agents on pancreas graft survival rates is universal or influenced by the duct management and recipient categories, is not clear, and will be the subject of future analyses.

\section{Preservation}

More than half of the pancreas grafts transplanted during the 1986-90 period were preserved in University of Wisconsin (UW) solution ( $\mathrm{n}=1111$ ), and the functional survival rate associated with its use was significantly higher $(p=0.001)$, than those for grafts stored in either Collins ( $n=695)$, plasma based $(n=177)$, or other solutions $(\mathrm{n}=75)$, at one year being $70 \%$ versus $56 \%, 55 \%$, and $49 \%$, 
respectively (Figure 8 ).

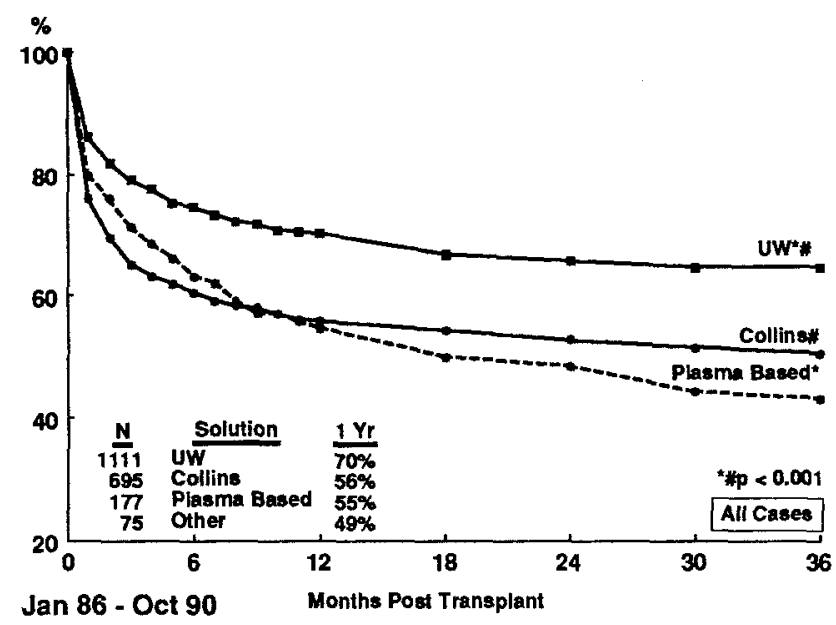

Fig.8. Pancreas graft functional survival rates for 1986-90 world cases according to preservation solution used

The technical failure rate for a pancreas graft stored in UW solution was $12 \%$, significantly lower $(\mathrm{p}<0.03)$ than the TF rate for grafts stored in Collins (21\%), plasma based (19\%), or other solutions $(31 \%)$. However, the lower TF rate is not the sole explanation for the better outcome for grafts preserved in UW solution, since even in the analysis of TS cases, UW ( $n=976)$ was associated with a significantly higher $(\mathrm{p}<0.001)$ graft functional survival rate than either Collins $(n=546)$, plasma $(n=144)$, or other solutions $(n=59)$, at one year $80 \%$ versus $69 \%, 67 \%$, and $59 \%$ respectively.

The proportion of grafts stored in UW solution increased toward the end of the 1986-90 period (from $7 \%$ for $1986-87$ to $75 \%$ for $1988-90$ cases). Thus, part of the differences in outcome with UW versus the other solutions may reflect the general trend for results to improve with time regardless of preservation solution used. However, in a separate analysis of $1988-90$ cases, the functional survival rate was higher for grafts stored in UW than other solutions. The distribution of UW solution within the various duct management and recipient categories could also influence the results, but in all the subanalyses, UW was associated with significantly higher graft survival rates than the other solutions. For example, for all BD cases, the one year functional survival rates for grafts stored in UW $(n=1000)$, Collins $(n=381)$, plasma based $(n=168)$, and other solutions $(n=8)$, were $72 \%, 57 \%$, $56 \%$, and $57 \%$, and the same relative differences were seen in the analysis of BD TS cases with one year graft functional survival rates of $81 \%(n=886), 67 \%(n=315)$, $67 \%(\mathrm{n}=138)$, and $57 \%(\mathrm{n}=8)$, respectively $(\mathrm{p}<0.001$ for UW versus Collins and plasma solutions in both analyses).

The other variable of interest in regard to pancreas graft preservation is the duration of cold storage. Sur- prisingly, up to 30 hours, the pancreas graft functional survival rates progressively improved with increasing storage times (Figure 9).

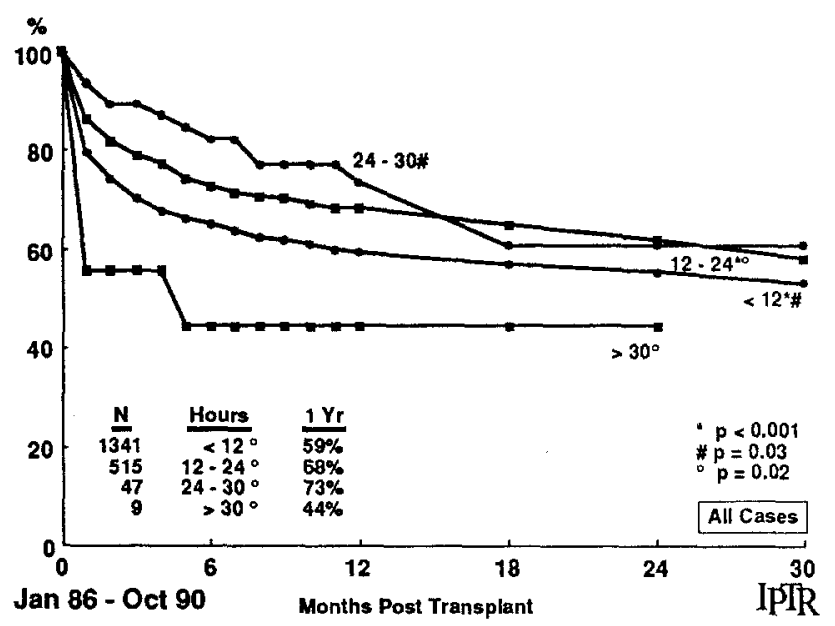

Fig.9. Pancreas graft functional survival rates for all 1986-90 world cases according to preservation time

This result may be due to the fact that longer storage times were used in a higher proportion of cases at the end than at the beginning of the 1986-90 period. Indeed, graft functional survival rates for grafts stored $24-30(n=47)$, or $12-24$ $(n=515)$ hours were both higher than for grafts stored $<12$ hours $(\mathrm{n}=1341)(\mathrm{p}<0.03)$, at one year being $73 \%, 68 \%$, and $59 \%$, respectively. The functional survival rate was also significantly higher $(\mathrm{p}<0.04)$ for grafts stored $24-30$ hours than for grafts stored $>30$ hours $(n=9)$ ( $44 \%$ at one year), but the difference in functional survival rates for grafts stored $<12$ or $>30$ hours were not significant. The storage time was unknown for $6.5 \%$ of the grafts $(n=146)$ and in these cases the one year functional survival rate was $71 \%(\mathrm{p}<0.05 \mathrm{vs}$ the $>30$ hour group).

The technical failure rate was higher for grafts stored $<12$ hours than those stored $12-24$, or $24-30$ hours, $18 \%$, $12 \%$, and $11 \%$ respectively, and the difference was significant $(p<0.01)$ for the $<12$ versus the $12-24$ hour groups. Again, the differences in technical failure rates are not solely responsible for the overall differences in outcome, since even in the analysis of technically successful cases the graft survival rates were higher in the 24-30 ( $n=42)$ and 12-24 hour $(n=455)$, than in the $<12$ $(\mathrm{n}=1095)$ hour storage groups, at one year being $80 \%$, $77 \%$, and $72 \%$ respectively ( $\mathrm{p}<0.04$ for the $12-24$ versus the $<12$ hour groups). For technically successful grafts stored $>30$ hours $(n=7)$, the graft survival rate was $57 \%$, not significantly different than the rates at the other storage times.

A possible explanation for the improved results with storage times between 12 and 30 hours as opposed to < 12 hours is the fact that storage times tend to be longer 
at the end than at the beginning of the 1986-90 period. However, UW solution was also used in a much higher proportion of the grafts stored for $>12$ hours $(78 \%)$ than $<12$ hours $(42 \%)$.

In a separate analysis of outcome according to preservation time for grafts stored in UW solution, the differences in graft functional survival rates were of a much smaller magnitude, although still significant for the $<12$ versus the $12-24$ hour storage group $(\mathrm{p}<0.03$ ) (Figure 10).

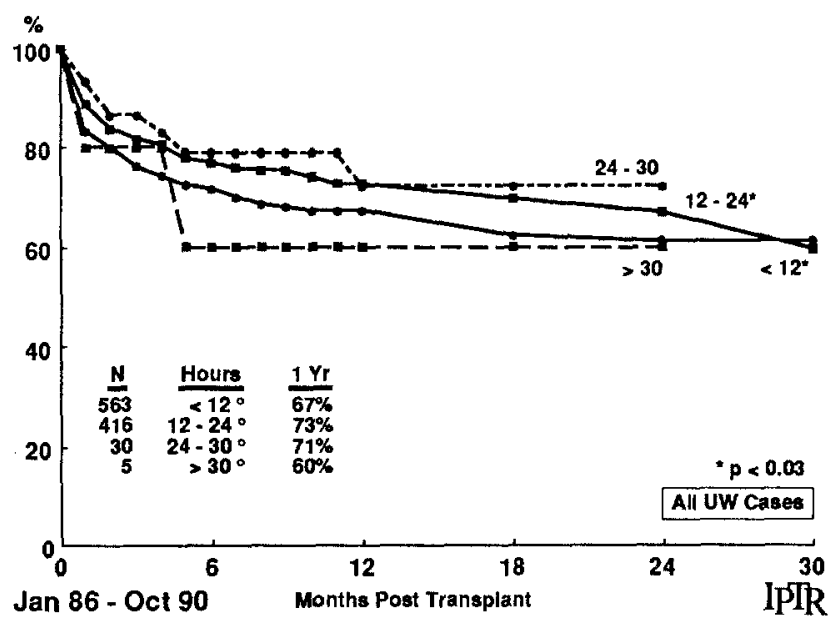

Fig.10. Pancreas graft functional survival rates for 1986-90 world cases according to preservation time in UW solution

For grafts preserved in UW solution during 1986-90, the one year functional survival rates were $67 \%$ in the $<12$ $(n=563), 73 \%$ in the $12-24(n=416), 71 \%$ in the $24-30$ $(n=30), 60 \%$ in the $>30$ hour $(n=5)$ storage groups, and $77 \%$ for UW stored grafts whose storage times were unknown ( $\mathrm{n}=97)$.

In an analysis of the best subgroup by duct management technique and recipient category, BDSPK transplants, there was absolutely no difference in outcome according to storage times in UW solutions, with one year pancreas graft functional survival rates in the $<12$ $(n=405), 12-24$ hour $(n=329), 24-30 \quad(n=21)$, and $>30$ $(\mathrm{n}=2)$ hours being $75 \%, 77 \%, 74 \%$, and $100 \%$, respectively. This data leaves no doubt that $U W$ is effective as a pancreas graft preservation solution for at least 30 hours. Not enough cases have been stored for $>30$ hours for a conclusion to be drawn.

\section{Comparison of Pancreas Transplant Outcome Ac- cording to Location}

Most pancreas transplants have been performed in North
America and Europe, and in this section the results from the two continents are compared to each other as well as to the results in other locations. Between January 1, 1986 and October 21, 1990, 1308 pancreas transplants were reported from North America, 743 from Europe, and 36 from other locations. Patient survival rates were not significantly different for recipients in North America and Europe (Figure 11), at one year being $90 \%$ and $88 \%$, respectively.

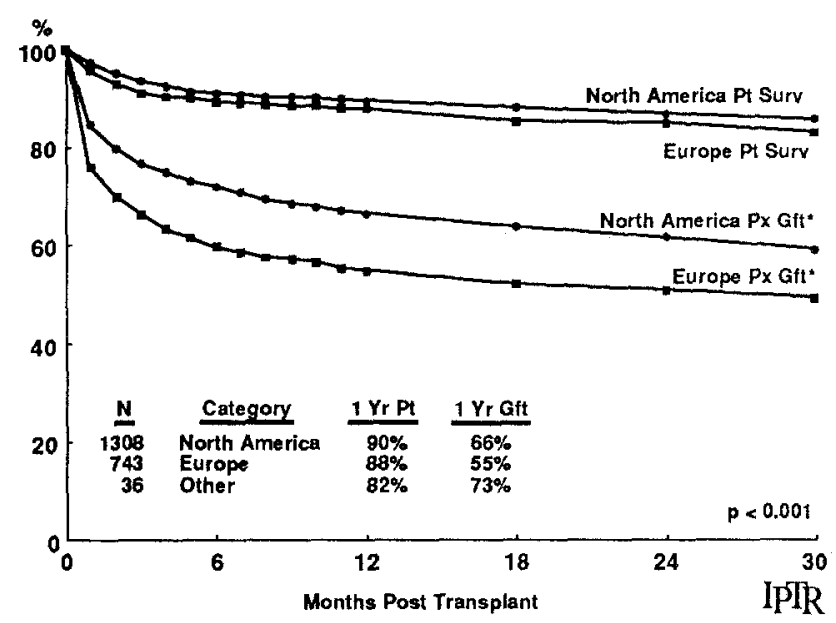

Fig.11. Patient and pancreas graft functional survival rates for $1986-90$ North America versus European cases

The survival of North American recipients was also significantly higher $(\mathrm{p}<0.05)$ than that of pancreas transplant recipients in non-European locations ( $82 \%$ survival at one year). Pancreas graft survival rates were also significantly higher $(p<0.001)$ in North America than in Europe, but neither differed significantly from the rate at other locations, at one year being $66 \%, 55 \%$, and $73 \%$, respectively. The same relative differences were seen in the analysis of technically successful cases, with one year functional survival rates being $77 \%$ in North America $(n=1127), 69 \%$ in Europe $(n=585)$, and $82 \%$ in other locations $(n=32)$ ( $p<0.001$ for North America versus Europe). The technical failure rates in North America, Europe and other locations were $14 \%, 21 \%$, and $11 \%$ ( $\mathrm{p}<0.001$ for North America versus Europe).

\section{Surgical Technique}

Almost all of the pancreas transplants performed in North America during the 1986-90 period were by the bladder-drainage technique $(n=1224,94 \%$ of the total for the continent), while in Europe less than half ( $42 \%$ ) were bladder-drained $(n=313)$. Even within the bladder-drainage technique category, graft functional survival rates were significantly higher $(p<0.001)$ in North 
America than in Europe (Figure 12),

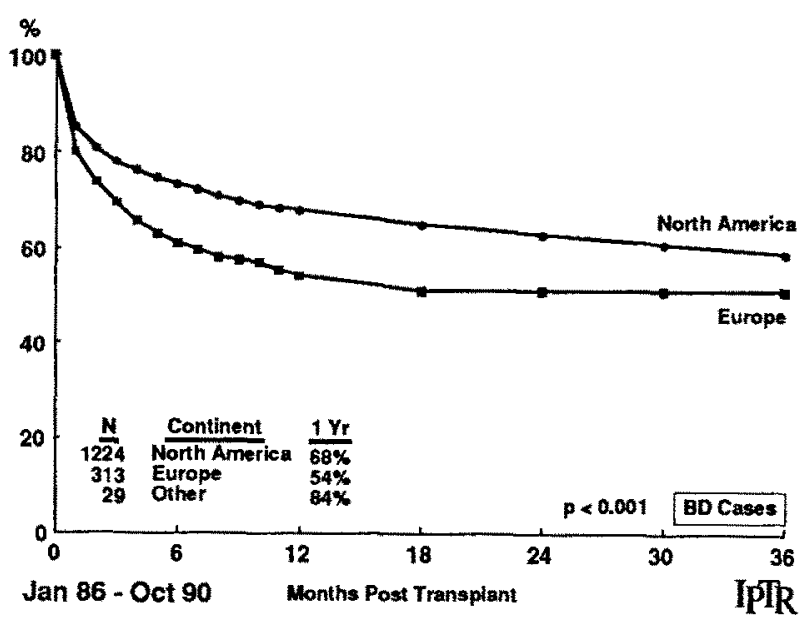

Fig.12. Bladder-drained pancreas graft functional survival rates for $1986-90$ North America versus European cases

$68 \%$ versus $54 \%$ at one year, and the same was true for bladderdrained pancreas transplants performed at other locations $(n=29)$, with the one year graft functional survival rate of $84 \%$ ( $p<0.01$ versus Europe). The technical failure rates for bladder-drained cases werenotsignificantly differentinNorth America, Europeand elsewhere $(13 \%, 17 \%$, and $3 \%$, respectively). In the analysis of TS $\mathrm{BD}$ cases, the graft functional survival rate was also significantly higher ( $p<0.001$ ) in North America than in Europe, being $78 \%$ versus $66 \%$ at one year. For TS BD cases at other locations $(n=28)$, the graft survival rate was almost significantly different than that of Europe ( $\mathrm{p}=0.07$ ), being $87 \%$ at one year.

The only other duct-management technique used in North America case during the 1986-90 period was intestinal drainage (ID, $n=63$ ) in one-twentieth of cases. This technique was used in more than one-seventh of European cases $(n=107)$. There was no difference in functional survival rates, for ID pancreas transplants performed in the two locations, at one year being $54 \%$ vs. $51 \%$, respectively, and $72 \%(n=47)$ vs. $63 \%(n=84)$ for TS cases (Figure 13).

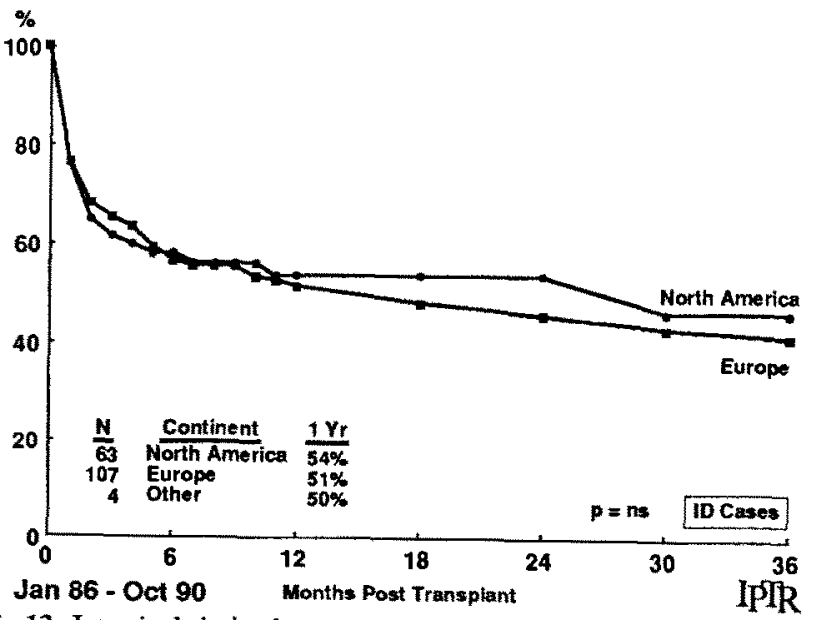

Fig.13. Intestinal drained pancreas graft functional survival rates for 1986-90 North America versus European cases
The technical failure rates for $\mathrm{ID}$ cases in North America and Europe were $28 \%$ and $25 \%$, not significantly different.

Duct injection was used exclusively in Europe $(n=319)$, with a one year graft functional survival rate of $56 \%$ for all cases and $73 \%$ for TS cases $(n=239)$. The technical failure rate for European DI cases was 25\%.

\section{Recipient Categories}

In both North America and Europe, as well as elsewhere, the majority of 1986-90 pancreas transplants were in the SPK category, comprising $75 \%, 85 \%$, and $78 \%$ of the total in the respective locations ( $n=986$ in North America; $n=630$ in Europe; and $n=78$ elsewhere). Pancreas transplants after a kidney comprised $14 \%$ of North American cases $(n=184)$, a significantly higher proportion $(\mathrm{p}<0.001)$ than the $5 \%$ in Europe $(n=39)$, while there were no cases in this category elsewhere. Pancreas transplants alone comprised $10 \%$ of cases in both North America $(n=133)$ and Europe $(n=71)$, while $22 \%$ of the cases elsewhere were in this category $(n=8)$. In all locations, the hierarchy seen in the world analysis was maintained, with graft functional survival rates being highest in the SPK and lowest in the PTA categories, while those in the PAK group were intermediate. Within each category, a comparison of outcome was made according to the location of the transplant.

In the SPK category, patient survival rates were not significantly different in the three locations, at one year $89 \%$ in North America, $87 \%$ in Europe, and $85 \%$ elsewhere. In contrast, pancreas graft survival rates for SPK cases were significantly higher $(p<0.001)$ in North America than in Europe, at one year being $74 \%$ versus $60 \%$, and the pancreas graft functional survival rate was also significantly higher $(p<0.02)$ for SPK cases performed elsewhere (84\% at one year) versus Europe (Figure 14).

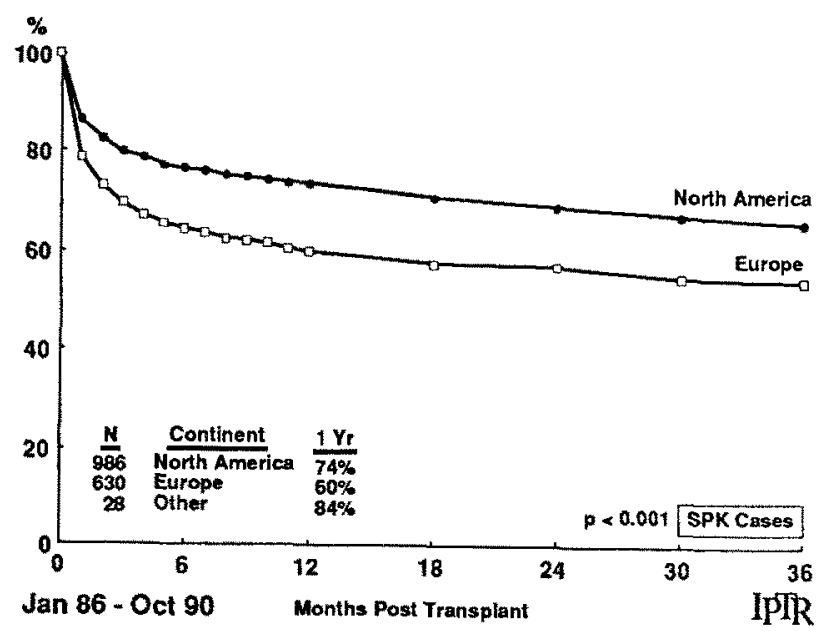

Fig.14. Pancreas graft functional survival rates for 1986-90 North America versus European simultaneous kidney transplant cases 
The TF rate for SPK cases was lower in North America than Europe (13\% versus 18\%, $p<0.003$ ), and was only $7 \%$ for other-located cases. However, this was not the explanation for the differences in outcome by location, since when TS cases were analyzed, the pancreas graft survival rates in the SPK category was significantly higher $(\mathrm{p}<0.001)$ in North America $(n=861)$ than in Europe ( $n=515)$, at one year being $84 \%$ and $73 \%$, respectively; the pancreas graft functional survival rates for TS SPK cases performed at other locations $(n=26)$ was also significantly higher $(p=0.05)$ than in Europe, at one year being $91 \%$.

In the PAK category, neither patient nor graft survival rates differed significantly between North America and Europe; at one year $93 \%$ and $96 \%$ of the recipients in the respective locations were alive, and $48 \%$ and $32 \%$ in the respectively, had a functioning pancreas (Figure 15).

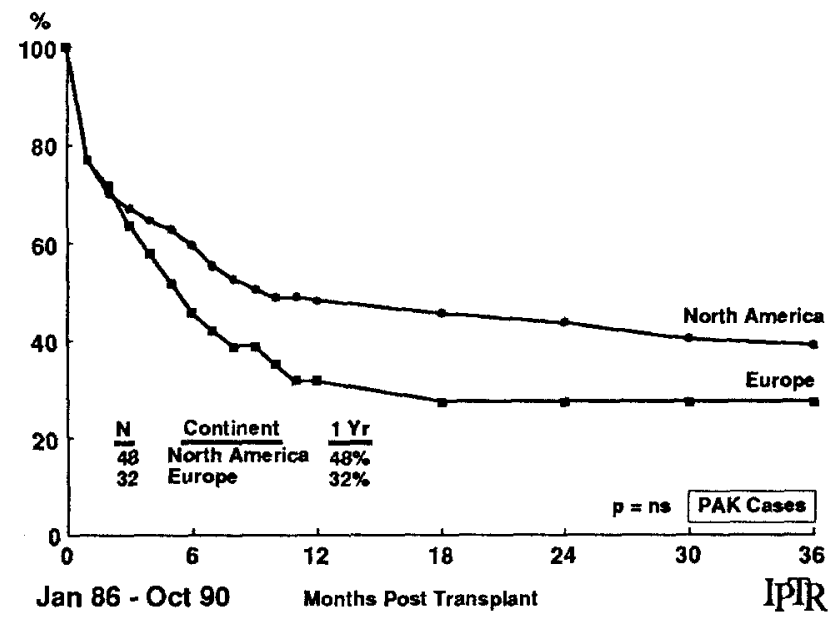

Fig.15. Pancreas after kidney graft functional survival rates for 1986-90 North America versus European cases

The TF rate for PAK cases was lower in North America (18\%) than in Europe (23\%), but the difference was not significant. Neither did the graft functional survival rates differ significantly between North America and Europe for TS PAK cases, at one year being $58 \%$ and $37 \%$ respectively.

In the PTA category, patient survival rates were similar in North America and Europe, at one year being $92 \%$ and $91 \%$ respectively while elsewhere the survival rate was significantly $(\mathrm{p}<0.05)$ lower $(73 \%$ at one year) than in North America. In contrast, PTA graft functional survival rates were significantly higher $(\mathrm{p}<0.05)$ in North America than in Europe or other locations, at one year being $46 \%, 22 \%$, and $38 \%$, respectively (Figure 16).

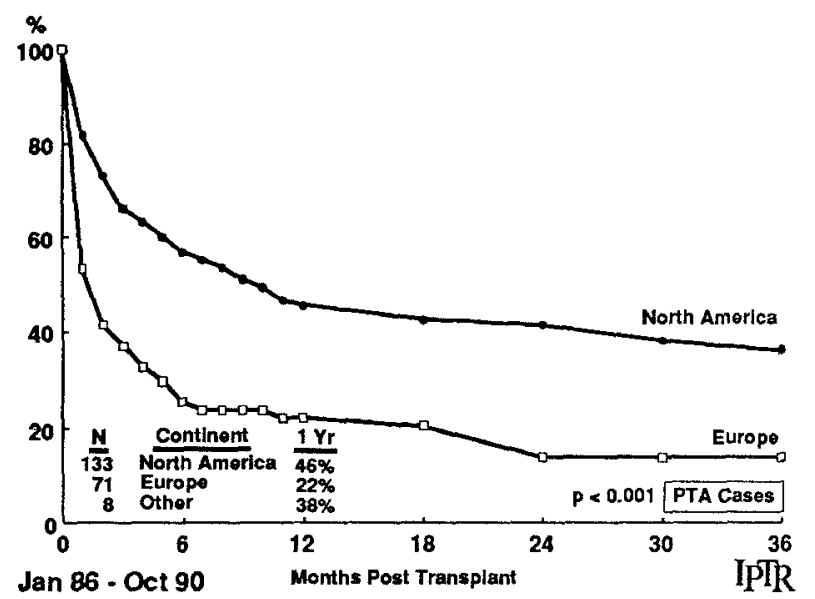

Fig.16. Pancreas transplant alone graft functional survival rates for $1986-90$ North America versus European cases

The TFrate forPTAcaseswas significantly lower $(\mathrm{p}<0.001)$ in North AmericathaninEurope (17\% versus $46 \%$ ), and was $25 \%$ for thecases performed elsewhere ( $p=n s$ versus North America and Europe). However, even for TS PTA cases the pancreas graft functional survival rate was significantly higher $(p<0.02)$ in North Americathan in Europe, at one year being $55 \%$ and $39 \%$, while the graft survival rate for TS PTA cases performed elsewhere did not differ significantly from either of the other locations, at one year being $50 \%$.

Theoutcomeaccording to locationineachrecipientcategory wasalso calculated for BD cases, but the resultsparalleled those obtained in the analysis of all cases and therefore are not shown. Thus, factors other than the differences in the proportional use of the various surgical techniques are responsible for the better results in North America.

\section{Kidney Graft Survival in SPK Recipients}

In the analysis of all 1986-90 SPK transplants, the kidney graft survival rate was significantly higher in North America than Europe, at one year being $83 \%$ versus $76 \%$. The kidney functional survival rate in SPK cases performed at other locations did not differ significantly from either North America or Europe, with $75 \%$ functioning at one year (Figure 17).

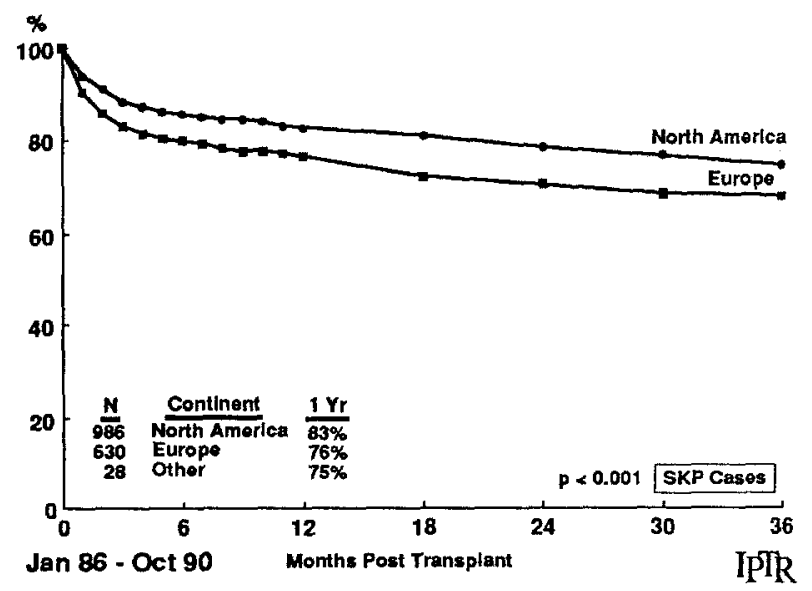

Fig.17. Kidney graft functional survival rates for 1986-90 North America versus European simulaneous pancreas transplant cases 
The one year kidney functional survival rate was also determined for SPK cases where the pancreas was TS and for cases in which the pancreas was a TF. For SPK cases in which the pancreas was TS, the kidney graft functional survival rate was significantly higher in North America than Europe, at one year being $86 \%$ versus $78 \%$, while there was no difference for either versus other located cases, with $80 \%$ functioning at one year. In contrast, for SPK cases where the pancreas was a TF, the kidney graft functional survival rates did not differ significantly between North America and Europe, at one year being $62 \%$ and $69 \%$ respectively. In both locations, the kidney graft functional survival rate was significantly higher for SPK cases in which the pancreas was TS than TF $(p<0.002)$.

\section{Preservation}

The proportion of pancreas grafts that were stored in UW solution during the $1986-90$ period was significantly higher $(p<0.01)$ in North America $(63 \%)$ than in Europe $(31 \%)$ or other locations $(41 \%)$. Collins solution was used to preserve $18 \%, 60 \%$, and $44 \%$ of the grafts at the respective locations. Plasma based solutions were used for $13 \%$ of the cases in North America, but for $<1 \%$ of cases elsewhere. Other solutions were used in $9 \%$ of European cases, but were rarely used elsewhere.

For grafts stored in UW solution, the graft functional survival rate was significantly higher $(p<0.001)$ in North America $(n=867)$ than in Europe $(n=229)$, at one year being $73 \%$ versus $57 \%$. In other locations, the results with UW solution $(n=15)$ were superb, with a one yeargraft functional survival rate of $100 \%$ ( $\mathrm{p}=0.053$ versus North America and $<0.01$ versus Europe). For grafts stored in Collins solution, there were no significant differences in outcome according to location, with one year graft functional survival rates in North America $(n=236)$, Europe $(n=443)$ and elsewhere $(\mathrm{n}=16)$ being $56 \%, 55 \%$, and $68 \%$. In North America, the one year functional survival rate for grafts stored in plasma based solutions $(n=173)$ was $55 \%$, and for the European cases stored in other solutions $(n=67)$ it was $51 \%$.

The technical failure rates were lower in North America than in Europe for grafts stored in either UW solution ( $11 \%$ versus $19 \%, \mathrm{p}<0.01$ ), or Collins solutions ( $20 \%$ versus $23 \%, \mathrm{p}=\mathrm{ns}$ ). In North America with plasma based solutions the TF rate was $19 \%$, and in Europe with other solutions it was $22 \%$. In the analysis of TS cases according to location and preservation solution used, the same relative differences were seen as in the analysis of all cases. With UW solution, graft functional survival rates were significantly higher in North America $(n=775)$ than Europe, at one year being $82 \%$ versus $71 \%(\mathrm{p}<0.001)$. For TS grafts stored in Collins solution, the one year graft functional survival rate was $68 \%$ in North
America $(\mathrm{n}=189), 69 \%$ in Europe $(\mathrm{n}=343)$, and $78 \%$ elsewhere $(n=14)$. In North America, the one year functional survival rate for TS grafts stored in plasma based solutions was $67 \%(n=140)$, and in Europe, the one year functional survival rate for TS grafts stored in other solutions was $62 \%(\mathrm{n}=52)$.

A much higher proportion of grafts were stored for longer than 24 hours in North America than Europe. In North America, only $50 \%$ of grafts were stored $<12$ hours $(n=649)$, while $35 \%$ were stored $12-24$ hours $(n=462)$, and $4 \%$ were stored between $24-30$ hours $(n=69)$. In contrast, in Europe, $89 \%$ of grafts were stored $<12$ hours ( $\mathrm{n}=662$ ), and only $6 \%$ were stored for $12-24$ hours $(\mathrm{n}=49)$, with none being stored for longer than this period. For grafts transplanted in other locations, $83 \%$ were stored $<12$ hours $(n=30)$, with the remainder stored from 12-24 hours.

At all storage times for which a comparison could be made, the graft functional survival rates were significantly higher $(p<0.01)$ for pancreas transplants performed in North America than Europe, at one year in the $12-24$ hour group being $63 \%$ versus $55 \%$, and in the 12-24 hour groups being $70 \%$ versus $55 \%$. For grafts stored 24-30 hours in North America the one year functional survival rate was $69 \%$. The TF rate was also lower in North America than in Europe at all storage times for which a comparison could be made, at $<12$ hours being $16 \%$ versus $21 \%(p<0.04)$ and at $12-24$ hours being $10 \%$ versus $24 \%(\mathrm{p}<0.01)$. In North America, the TF rate after 24-30 hours of storage was $12 \%$. However, in the analysis of TS cases, a significant difference $(p<0.03)$ between North America and Europe was seen only at the $<12$ hour storage time, with one year graft functional survival rates being $76 \%$ in North America $(n=544)$ and $69 \%$ in Europe $(n=524)$. After 12-24 hours storage the one year graft functional survival rate was $77 \%$ in North America $(n=414)$ and $76 \%$ in Europe $(n=37)$. For TS grafts stored $24-30$ hours in North America $(n=49)$, the one year graft functional survival rate was $77 \%$.

\section{Multivariate Analyses}

In order to determine whether the factors found to be associated with significantly higher graft survival rates in the univariate analysis were an artifact of association with other variables, a Cox proportional hazard analysis was performed on 1986-90 cases in which duct management, recipient category, HLA-DR mismatching (MM), immunosuppression with anti- $\mathrm{T}$ cell agents, preservation solution, duration of preservation, location of the transplant, and year of the transplant were the variables. The analysis was performed on all cases as well as on technically successful cases. 
The result of the analysis on all cases is shown in Table 1:

Table 1. Relative risk (RR) of graft loss according to Cox multivariate analysis of all 1986-90 world pancreas transplants

\begin{tabular}{lrc}
\hline Variable & Zeta $^{*}$ & Relative Risk $^{\mathbf{b}}$ \\
\hline Retransplant vs primary & 1.62 & 1.24 \\
BD vs ID & -1.57 & 0.83 \\
ED vs ID & -1.00 & 0.87 \\
SPK vs PTA* & -7.81 & 0.43 \\
PAK vs PTA & -1.35 & 0.84 \\
DRMM1, 2 vs 0** & 1.98 & 1.19 \\
ALG or OKT3 vs None & -1.52 & 0.89 \\
ALG \& OKT3 vs None & 0.72 & 1.25 \\
Collins Sol vs Other & -0.25 & 0.97 \\
UW Sol vs Other & -0.99 & 0.88 \\
Pres 24-30 Hrs vs <24 Hrs & -1.27 & 0.71 \\
Pres > 30 Hrs vs <24 Hrs & 0.69 & 1.37 \\
Year 1989-90 vs 1986-88* & -2.70 & 0.76 \\
Europe vs NA* & 3.01 & 1.34 \\
& & \\
\hline${ }^{*}$ < $<.01$ & \\
** $<$ <0.05 & & \\
a Coefficient/Standard error & \\
b Exponential (coefficient) &
\end{tabular}

In the Cox analysis of all cases, neither retransplantation nor duct management technique had a significant impact on graft survival rates. However, recipient category was very important, with transplantation of the kidney simultaneous with a pancreas significantly lowering the risk of pancreas graft loss. In accordance with previous univariate analyses (but not in the current one), risk for graft loss was significantly higher in the combined 1 or $2 \mathrm{DR}$ mismatch than the $0 \mathrm{DR}$ MM group. (For the $1 \mathrm{DR}$ MM alone vs the $0 \mathrm{DR}$ MM group the RR was 1.17 $[\mathrm{p}=0.09]$ and for the $2 \mathrm{DR} M \mathrm{MM}$ alone group vs the $0 \mathrm{DR} \mathrm{MM}$ group the $R R$ was 1.21 [p=0.051].) Use of anti-T cell agents for induction immunosuppression, preservation solution, and duration of storage did not have a significant impact on outcome in the Cox analysis, but year of transplant did. The risk of graft loss was significantly lower for transplants performed in 1989 . 90 than in 1986-88. Finally, the location of the transplant had a significant impact; inexplicably, the risk for pancreas graft loss was higher in Europe than in North America.

An identical Cox analysis was performed for technically successful(TS) cases, and 3 of the 4 factors identified as significant in the analysis of all cases persisted as significant in this analysis. Again, duct management did not have an impact, but recipient category did, with the $R R$ for loss of a TS pancreas graft in SPK cases being 0.35 vs the other categories $(p<0.001$ ). Year of transplant was significant, with a RR of 0.72 for 198990 vs $1986-88$ cases $(p<0.03)$, and location also remained significant with a relative risk (RR) of 1.52 for Europe vs North American TS cases $(p<0.002)$. The one factor significant in the Cox analysis of all cases that was not in the analysis of TS cases, was the degree of HLA-DR mismatching; the RR was 1.26 for 2 DR MM ( $p=0.11)$ and 1.06 for 1 DR MM ( $p=0.8)$ TS grafts (the coefficients were toodisparate toallow fora validcombined analysis). On the other hand in the analysis of TS cases, one factor emerged as significant that was not significant in the analysis of all cases, use of either ALGorOKT3 for prophylactic immunosuppression. The RR for loss of TS grafts in recipients treated with one or the other of the anti-T cell agents was 0.80 $(\mathrm{p}<0.05)$.

In the overall univariate analysis, the incidence of technical failure was significantly lower in North America than in Europe. To determine whether location or other variables truly were independent risk factors, a logistic regression analysis was performed, with technical failure being the dependent variable. In this analysis, location did not have a significant impact on the risk of technical failure ( $\mathrm{p}=0.16$ for North America vs Europe). Year of transplant (1986-88 vs 1989-90), degree of DR mismatching and use of anti-T cell agents for prophylactic immunosuppression also did not have a significant impact on the incidence of technical failure, but retransplantation, duct management technique, recipient category, preservation solution, and storage time did (Table 2).

Table 2. Factors significantly increasing the relative risk (RR) of technical failure for 1986-90 world pancreas transplants

\begin{tabular}{lll}
\hline Variable & RR & p-value \\
\hline $\begin{array}{l}\text { ReTx vs Primary } \\
\text { Duct mgmt technique }\end{array}$ & 1.34 & $<0.0001$ \\
ED vs BD & 1.34 & $<0.0001$ \\
DI vs BD & 1.39 & \\
Recipient category & & $<0.0001$ \\
$\quad$ PAK vs SPK & 1.28 & \\
PTA vs SPK & 1.36 & $<0.02$ \\
Preservation solution & & \\
$\quad$ Other vs UW & 1.14 & $<0.003$ \\
$\quad$ Collins vs UW & 1.19 & \\
Preservation Time & & \\
$24-30$ vs $<24$ Hrs & 1.26 & \\
$>30$ vs $<4$ Hrs & 1.41 &
\end{tabular}

Compared to the alternatives, the RR of pancreas graft technical failure was significantly lower with bladder-drainage, simultaneous transplantation of a kidney, preservation in UW solution, and storage for $<24$ hours. Some of the factors identified as predisposing to technical failure by logistic regression analysis were the same as those that increase the RR for graft loss in the Cox analysis of all cases, while others were not. All causes of graft loss were included in the Cox analysis, and there is no inherent reason why the risk of technical failure should bear a relation to the risk of graft loss for immunological or other reasons. On the other hand, it is possible that some of the early losses interpreted as TF are actually rejections causing secondary thrombosis, an event more likely in the PTA and PAK than in the SPK categories.

Likewise, the significance of some of the differences seen in the univariate analyses were confirmed in the multivariate analyses, while others were not, showing the need to consider the 
influence of confounding variables when interpreting the results. For example, duct management technique was not a significant factor in the Cox analysis, but graft survival was significantly higher for $\mathrm{BD}$ grafts in the univariate analyses and $\mathrm{BD}$ grafts were also less likely to fail for technical reasons in both the univariate and the logistic regression analyses. The benefit of a simultaneous kidney transplant on pancreas graft survival rates was seen in the univariate and both multivariate analyses. In the Cox analysis of all cases, DR mismatching had a significant impact on outcome, but in the univariate analysis, the degree of DR mismatching was significant only in the analysis of PTA cases. Inexplicably, in the analysis of TS cases DR mismatching was not significant. Preservation solution had a significant effect on outcome in the univariate analysis but not in the Cox analysis; however, UW solution was associated with significantly fewer technical failures than other solutions in the logistic regression analysis. Conversely, preservation time did not significantly effect outcome in either the univariate or $\mathrm{Cox}$ analyses, but TF rates were significantly higher for grafts stored $>24$ hours than $<24$ hours in the logistic regression analyses. Year of transplant was a significant factor in outcome in both the univariateandCox analyses, butdidnothaveasignificantimpacton the TFrate. Location hada significanteffectonoutcome on both the univariate and Coxanalyses, butaccording tothe logisticregression analysis did not influence the TF rates.

\section{Discussion}

As in previous analyses of Registry data, the results of pancreas transplantation continue to improve and application continues to increase, particularly in diabetic uremic recipients of kidney transplants. Bladder-drainage was used more than any other duct management technique, had the lowest technical failure rate, and in a univariate analysis was associated with a significantly higher graft functional survival rate than the other techniques. However, in the Cox multivariate analysis, there were no significant differences in outcome according to duct management techniques. Most pancreas transplants are performed simultaneous with a kidney, and the benefit conferred by having a kidney from the same donor tomonitor for rejection may override any advantage that one duct management technique has over another. The dominant effect of kidney association and the lack of a significant influence of duct management on outcome is consistent with a previous multivariate analysis of Registry data (Viste et al. 1990).

Pancreas grafts can be preserved for longer than 30 hours, but logistic regression analysis shows that preservation for $>24$ hours predisposes to technical failure. The technical failure rate is lower for grafts stored in UW than other solutions. On univariate analysis, the use of UW was associated with a higher pancreas graft functional survival rate than the use of other solutions. However, in the Cox multivariate analysis, UW solution did not emerge as having a significant impact, most likely because UW was predominantly used in the latter portion of the 1986-90 period, and year of transplant was a significant independent variable, with cases performed in 1989-90having a significantly better outcome than those performed in 1986-88.

Immunological factors had less of an impact on outcome in this than in previous Registry analyses. Grafts from donors mismatched for $0 \mathrm{DR}$ antigens with the recipient had a significant better outcome in the overall analysis than those mismatched for 1 or 2DR antigens, but this effect was not seen in the Cox analysis of TS cases. In the univariate analysis, a significant effect of DR mismatching was seen only in the PTA cases.

Recipients of ALG or OKT 3 for prophylactic immunosuppression had significantly higher pancreas graft survival rates than those who did not. However, in the multivariate tests, the impact of ALG or OKT3 on outcome was significant only in the Cox analysis of TS cases.

An unexplained finding was the significantly higher graft survival rate for pancreas transplants in North America than in Europe. The difference was confirmed as real in the Cox multivariate analyses of all cases and of TS cases, and could not be attributed to differences in the technical failure rates according to logistic regression analysis. Future analyses will have to address other variables that might account for the differences in outcome between the two continents. Nevertheless, in all locations pancreas graft survival rates are within the range of those for other solid organ transplants, and there is no evidence that the results have plateaued.

Acknowledgments. The Intemational Pancreas Transplant Registry is supported by NIH contract DK-9-2244-Mod. 4 from the National Institute of Health. A portion of the data collected on U.S. cases was supplied by a subcontract with the United Network forOrgan Sharing. LeAnne Schauerprepared the manuscript and Kristin Lundin prepared the figures. Walter Schmidt assisted with the statistical analyses.

\section{References}

Cox DR (1972) Regression models and life tables. J Roy Stat B 34, pp 187 220

Gehan E (1965) A generalized Wilcoxin comparing arbitrarily singly sensored samples. Biometrika 52:203

Lemeshow S, Hosmer DW (1984) Estimating odds ratios with categorically scaled covariates in multiple logistic regression analysis. $J$ Epidemiol 119:147-151

Squifflet JP, Moudry K, Sutherland DER (1988) Is HLA matching relevant in pancreas transplantation? Transpl Inil 1:26-29

Sutherland DER, Chow SY, Moudry-Munns KC (1989) Intemational Pancreas Transplant Registry Report-1988. Clin Transpl 3:129-149

Sutherland DER, Moudry-Munns KC, Gillingham K (1990) Pancreas trans plantation: Report from the Intemational Registry and a preliminary analysis of United States results from the new United Network for Organ Sharing (UNOS) Registry. In Clinical Transplants-1989, Terasaki P (ed), UCLA Tissue Typing Laboratory, Los Angeles, pp 19-43

Viste, A, Moudry-Munns K, Sutherland DER (1990) Prognostic risk factors for graft failure following pancreas transplantation: results of multivariate analysis of data from the Intemational Pancreas Transplant Registry. Transpl Int 3:98-102

Dr. David Sutherland

UMHC Box 280

420 Delaware St. SE

Minneapolis, Minnesota 55455

USA 